\title{
Weak and Strong Computational Creativity
}

\author{
Mohammad Majid al-Rifaie and Mark Bishop
}

\begin{abstract}
In the spirit of Searle's definition of weak and strong artificial intelligence, this paper presents a discussion on weak computational creativity in swarm intelligence systems. It addresses the concepts of freedom and constraint and their impact on the creativity of the underlying systems. An analogy is drawn on mapping these two 'prerequisites' of creativity onto the two well-known phases of exploration and exploitation in swarm intelligence algorithms, followed by the visualisation of the behaviour of the swarms whose performance are evaluated in the context of arguments presented. The paper also discusses that the strong computational creativity is presented in ways emphasising that genuine creativity implies 'genuine understanding' and other cognitive states, along with autonomy - asserting that without 'Strong Embodiment', computational systems are not genuinely autonomous.
\end{abstract}

\section{Introduction}

In recent years, studies of the behaviour of social insects (e.g. ants and bees) and social animals (e.g. birds and fish) have proposed several new metaheuristics for use in collective intelligence resulting from social interaction.

Among the many works in the fields are research on swarm painting (e.g. [26, $8,37,38]$ ), ant colony paintings (e.g. [21, 25, 34]) and other multi-agent systems (e.g. RenderBots [32] and the particle-based non-evolutionary approach of Loose and Sketchy Animation [15]).

In most of the swarm-based work mentioned above (e.g. [26, 8, 37, 38, 21]), the painting process does not re-work an initial drawing, but rather focuses on presenting "random artistic patterns", somewhere between order and chaos [38]. Other

Mohammad Majid al-Rifaie

Goldsmiths, University of London, London SE14 6NW, e-mail: m.majid@gold.ac.uk

Mark Bishop

Goldsmiths, University of London, London SE14 6NW, e-mail: m.bishop@gold.ac.uk 
classes of research (e.g. by Schlechtweg et al. [32] and Curtis [15]) are based on reworking an initial drawing. There is a significant number of related papers in the area of non-photorealistic rendering; particularly, many papers approach drawing and painting using the optimisation framework (where optimisation and generative techniques are utilised an artistic context). Furthermore, particles have been used for stippling and other aesthetic styles in numerous papers. Turk and Bank's work [36] is an early example of optimising particle positions to control a stroke-based rendering. Hertzmann [22] optimised a global function over all strokes using a relaxation approach. In one of his works, Collomosse [14] used a global genetic algorithm to define a rendering algorithm. More recently, Zhao et al. [41] deployed an optimisation-based approach to study the stroke placement problem in painterly rendering, and presented a solution named stroke processes, which enables intuitive and interactive customisation of painting styles.

This work is an extension of ideas first presented at the Computing and Philosophy symposium at AISB 2011 [2] and subsequently published in the Cognitive Computation journal [6]. In the work discussed herein the impact of freedom and constraint on the concept of 'creativity' is discussed, followed by a discussion on the creativity of swarm intelligence systems. This paper also addresses the thorny issue of 'Weak' verses 'Strong' computational creativity.

\section{ON ART, FREEDOM AND CREATIVITY}

For many years there has been discussions on the relationship between art, creativity and freedom; a debate elegantly encapsulated in the famous German prose by Ludwig Hevesi at the entrance of the Secession Building in Vienna:

"Der Zeit ihre Kunst

Der Kunst ihre Freiheit"

That is: "To Time its Art; To Art its Freedom".

which, centuries after, resonates an earlier observation from Aristotle (384-322 BCE) [18] emphasising the importance of freedom, giving rise to further exploration of areas otherwise left untouched (here, having "a tincture of madness") in presenting a creative act.

"There was never a genius without a tincture of madness."

On the other hand Margaret Boden, in [9], more recently argues that creativity has an ambiguous relationship with freedom:

"A style is a (culturally favoured) space of structural possibilities: not a painting, but a way of painting. Or a way of sculpting, or of composing fugues .. [] .. It's partly because of these [thinking] styles that creativity has an ambiguous relationship with freedom." 
Considering the many factors constituting the evaluation of what is deemed 'creative', raises core issues regarding how humans evaluate creativity; their aesthetic capacity and potentially that of other animals (e.g. as exhibited in, say, mateselection). Galanter [19] suggests that perhaps the 'computational equivalent' of a bird or an insect (e.g. in evaluating mate selection) is all that is required for [computational] aesthetic evaluation:

"This provides some hope for those who would follow a psychological path to computational aesthetic evaluation, because creatures with simpler brains than man practice mate selection."

In this context, as suggested in [16], the tastes of the individual in male bowerbirds are made visible when they gather collections of bones, glass, pebbles, shells, fruit, plastic and metal scraps from their environment, and arrange them to attract females [10]:

"They perform a mating dance within a specially prepared display court. The characteristics of an individual's dance or artefact display are specific to the species, but also to the capabilities and, apparently, the tastes of the individual."

However the question of whether 'mate selection behaviour in animals implies making a judgement analogous to aesthetic judgement in humans' is perhaps (pace Nagel's famous discussion 'What is it like to be a bat?' [27]) a fundamentally unanswerable question.

In contrast, the role of education (or training) in recognising 'good' and 'bad', 'creative' and 'non-creative' has been experimentally probed. A suggestive study investigating this topic by Watanabe [39] gathers a set of children's paintings, and then adult humans are asked to label the "good" from the "bad". Pigeons are then trained through operant conditioning to only peck at good paintings. After the training, when pigeons are exposed to a novel set of already judged children's paintings, they show their ability in the correct classification of the paintings.

This emphasises the role of learning training and raises the question on whether humans are fundamentally trained (or "biased") to distinguish good and/or creative work.

Another tightly related topic to swarm intelligence in this context is the creativity of social systems. Bown in [11] indicates that our creative capabilities are contingent on the objects and infrastructure available to us, which help us achieve individual goals, in two ways:

"One way to look at this is, as Clark does [13], in terms of the mind being extended to a distributed system with an embodied brain at the centre, and surrounded by various other tools, from digits to digital computers. Another way is to step away from the centrality of human brains altogether and consider social complexes as distributed systems involving more or less cognitive elements."

Discussion on creativity and the conditions which make a particular work creative, have generated heated debate amongst scientists and philosophers for many years [31]; for a theoretical review on 'conditions of creativity'; the 'systems' view of creativity; cognitive approaches, etc. see also [35]. Although this article does not 
aim to resolve any of these issues (or even suggest that the presented work strongly fits and endorses the category of the 'computationally creative realm'), we investigate the performance of a swarm intelligence sketching system which, we suggest, highlights core issues inherent in exploring conceptual/artistic space(s).

\section{Creativity in Swarms}

\subsection{Freedom vs. Constraint}

Freedom and constraint have been at the core of several definitions for creativity. Philip Johnson-Laird in his work on freedom and constraint in creativity [23] states:

“... for to be creative is to be free to choose among alternatives .. [] .. for which is not

constrained is not creative."

In swarm intelligence systems, the two phases of exploration and exploitation introduce the freedom and control the level of constraint. Pushing the swarms towards exploration, freedom is boosted; and by encouraging exploitation, constraint is more emphasised. Finding a balance between exploration and exploitation has been an important theoretical challenge in swarm intelligence research and over the years many hundreds of different approaches have been deployed by researchers in this field. In the presented work, two swarm intelligence algorithms are deployed: the algorithm which is responsible for the "intelligent" tracking of the line drawing is Particle Swarm Optimisation (PSO) [17, 24]. This well-known algorithm, which mimics the behaviour of birds flocking, has an internal mechanism of balancing off the exploitation and exploration phases. However due to the weakness of the exploration in this algorithm, our system also deploys another nature inspired algorithm to overcome this weakness, Stochastic Diffusion Search (SDS) [1], which mimics the behaviour of one species of ants (Leptothorax acervorum) foraging. Therefore, exploration is promoted by utilising the SDS algorithm, whose impact on different swarm intelligence algorithms has been scientifically reported using various measures and statistical analysis in several publications (e.g. [7, 3, 5, 4]).

In the visualisation, the swarms are presented with a set of points (which constitute a line drawing - see Fig. 1) and are set to consider these points (one at a time) as their global optimum. In other words, the global optimum is dynamic, moving from one position to another and the swarms aim to converge over this dynamic optimum (Fig. 2).

As stated in the introduction, there have been several relevant attempts to create creative computer generated artwork using Artificial Intelligence, Artificial Life and Swarm Intelligence. Irrespective of whether the swarms are considered genuinely creative or not, their similar individualistic approach is not totally dissimilar to those of the "elephant artists" [40]:

"After I have handed the loaded paintbrush to [the elephants], they proceed to paint in their own distinctive style, with delicate strokes or broad ones, gently dabbing the bristles on the 
paper or with a sweeping flourish, vertical lines or arcs and loops, ponderously or rapidly

and so on. No two artists have the same style."

Similarly if the same line drawing (see Fig. 1) is repeatedly given to the swarms, the output sketches (e.g. Fig 2) made by the swarms, are never the same (see Fig. 4 to compare different sketches). In other words, even if the swarms process the same input several times, they will not make two identical sketches; furthermore, the outputs they produce are not merely randomised variants of the input. In order to demonstrate this claim qualitatively in an experiment, the output of the swarmbased system is compared against a simple randomised tracing algorithm, where each point in the line drawing could be surrounded with lines at a random distance and direction.

In Fig 3, only PSO algorithm is used to produce the sketch. This experiment is run in order to highlight the impact of removing exploration (i.e. 'freedom') which is otherwise induced by the SDS algorithm.

\subsection{Swarmic Freedom versus Random Freedom}

This part presents an experiment with the goal of contrasting the behaviour of the swarms to that of a group of random agents. In this experiment, the freedom of the swarm (i.e. Swarmic Freedom) is maintained by the swarm intelligence algorithms used in the system, whereas the freedom of the agents in the randomised algorithm is controlled by what we call the Random Freedom. These definitions are utilised here to highlight the potential of the swarms in exhibiting computational creativity.

The sketches in Fig. 5 (top and middle) show two outputs from a simple randomised algorithm when configured to exhibit limited 'random' variations in its behaviour (i.e. there is only small random distance and direction from the points of the original line drawing); comparing the two sketches, we note a lack of any significant difference between them. Furthermore, when more 'freedom' is granted to the randomised algorithm (by increasing the range in the underlying random number generator, which allows the technique to explore broader areas of the canvas), the algorithm soon begins to deviate excessively from the original line drawing. For this reason such randomisation results in a very poor - low fidelity - interpretation of the original line drawing (Fig. 5-bottom). In contrast, although the agents in the swarms are free to access any part of the canvas, the swarm-control mechanism (i.e. Swarm Freedom) naturally enables the system to maintain recognisable fidelity to the original input. In the randomised algorithm, contra the swarms system, it can be seen that simply by giving the agents more randomised behaviour (Random Freedom), they fail to produce more 'creative sketches'.

The Swarmic Freedom or 'controlled freedom' (or the 'tincture of madness') exhibited by the swarm algorithms (induced by the stochastic side of the algorithms) is crucial to the resultant work and is the reason why having the same line drawing does not result in the system producing identical sketches. This freedom emerges, among other influencing factors, from the stochasticity of the SDS algorithm in 
picking agents for communication, as well as choosing agents to diffuse information; the tincture of madness in the PSO algorithm is induced via its strategy of spreading the particles throughout the search space as well as the stochastic elements in deciding the next move of each particle.

In other words, the reason why the swarm sketches are different from the simple randomised sketches, is that the underlying PSO flocking component-algorithm constantly endeavours to accurately trace the input image whilst the SDS foraging component constantly endeavours to explore the wider canvas (i.e. together the two swarm mechanisms ensure high-level fidelity to the input without making an exact low-level copy of the original line drawing). Although the algorithms (PSO and SDS) are nature-inspired, we do not claim that the presented work is an accurate model of natural systems. Furthermore, whilst designing the algorithm there was no explicit 'Hundertwasser-like' attempt [30] by which we mean the stress on using curves instead of straight lines, as Hundertwasser considered straight lines not nature-like and tried not to use straight lines in his works to bias the style of the system's sketches.

\section{Weak vs. Strong Computational Creativity}

Before approaching the topic of weak or strong computational creativity, the difference between weak and strong AI is highlighted. In strong AI, the claim is that

Fig. 1 This figure shows a series of points that make a line drawing; sample line drawing after one of Picasso's sketches. 
Fig. 2 A sketch produced by the swarms, using both SDS and PSO algorithms.

Fig. 3 A sketch produced by the swarms without SDS exploration.
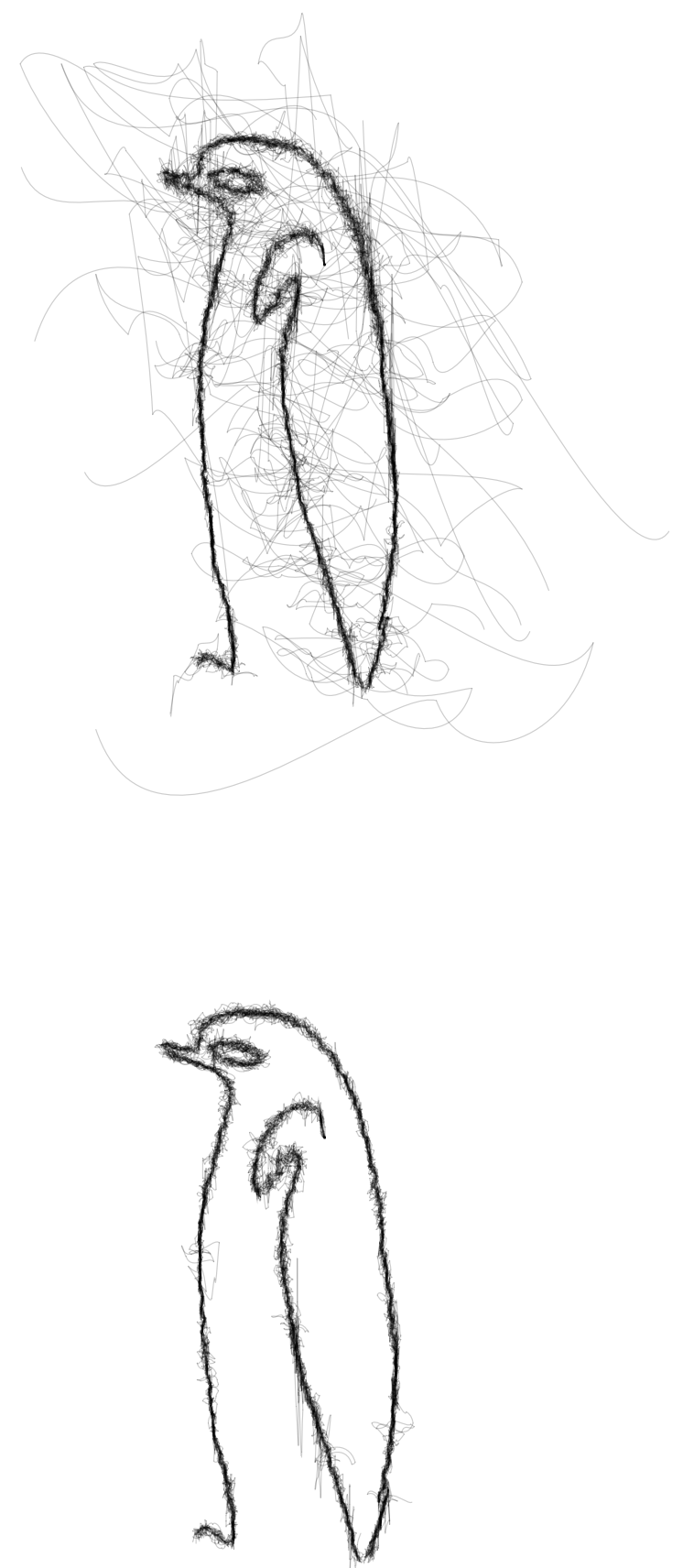
Fig. 4 Different sketches of the swarms off a single line drawing.

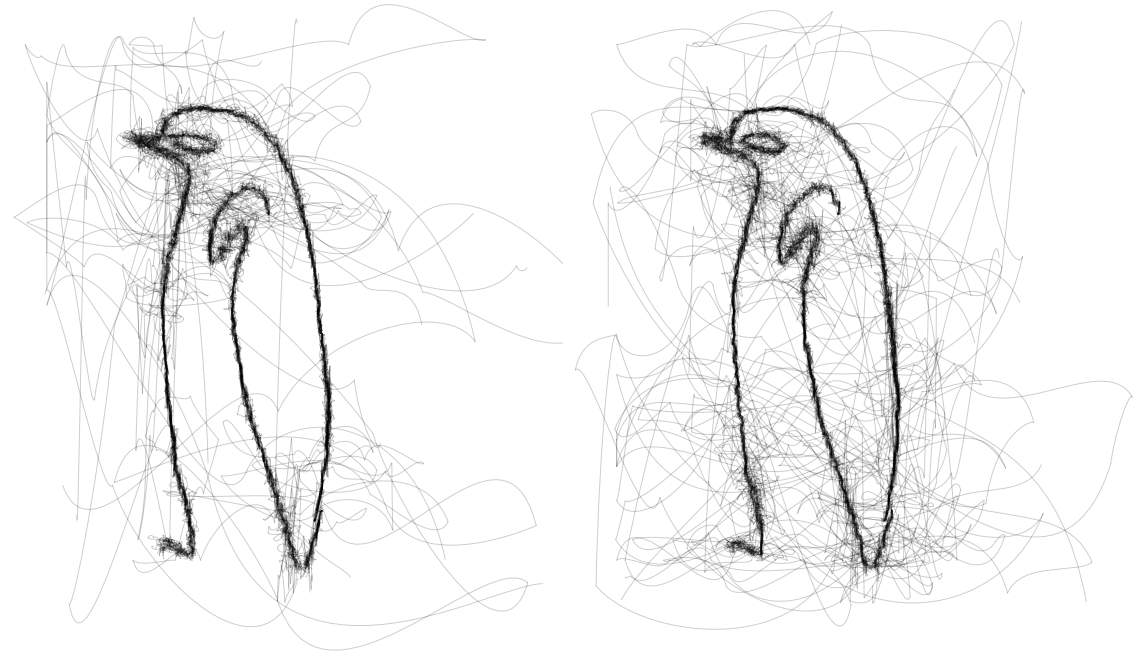

machines can think and have genuine understanding and other cognitive states (e.g. "suitably programmed machines will be capable of conscious thought" [12]); weak $\mathrm{AI}$, in contrast, does not usually go beyond expecting the simulation of human intelligence. I.e. instantiating genuine "understanding" is not the primary concern in weak AI research.

An analogy could be drawn to computational creativity, extending the notion of weak AI to 'weak computational creativity', which does not go beyond exploring the simulation of human creativity; emphasising that genuine autonomy and genuine understanding are not the main issues in conceptualising weak computationally creative systems. Conversely in 'strong computational creativity', the expectation is that the machine should be autonomous, creative, have 'genuine understanding' and other cognitive states.

The concept of 'Strong AI' - instantiating a computational machine with genuine understanding - has provoked many critics, among whom John Searle made perhaps the most famous attack with his Chinese Room Argument (CRA) [33] (for comprehensive discussion see [29]). The central claim of the CRA is that computations alone cannot in principle give rise to understanding, and that therefore computational theories of mind cannot fully explain human cognition. More formally, Searle stated that the CRA was an attempt to prove that syntax (rules for the correct formation of sentences:programs) is not sufficient for semantics (understanding). Combining this claim with those that programs are formal (syntactical), whereas minds have semantics, led Searle to conclude that programs are not minds.

But then it is equally clear that Searle accepts that there is no boundary on a fundamental level to the idea that a machine can think as in Minds, Brains and Pro- 
Fig. 5 The sketches of the swarms with random behaviour: This figure shows the sketches made with a simple randomised tracing algorithm, using random distance and direction from the lines of the original line drawing. The first two sketches (top and middle) use the same random distance (e.g. $d$ ) and the bottom sketch uses the random distance of $d \times 6$.
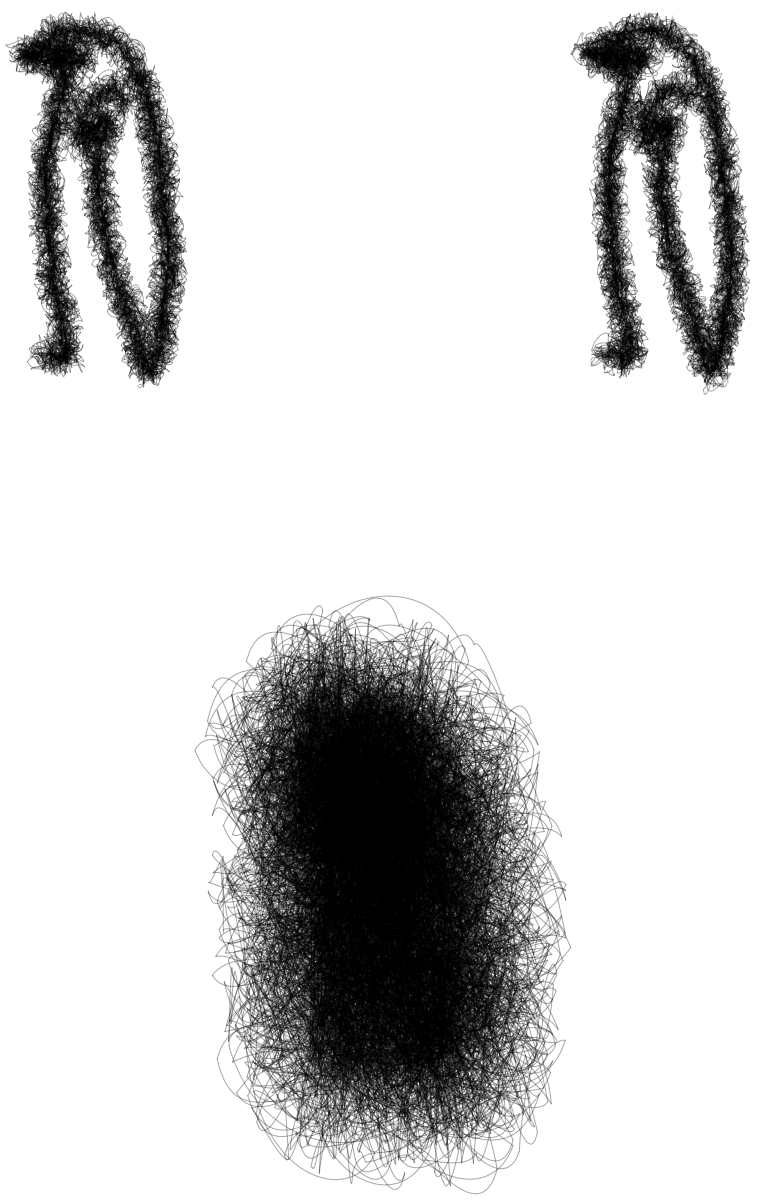
grams (MBP) Searle [33], in reply to the inquiry 'Can a machine think?", expressly states that the response is clearly 'yes', as we are precisely such machines.

Clearly Searle did not intend the CRA to target machine intelligence per se, but rather any form of artificial intelligence according to which a machine could have genuine mental states (e.g. understanding Chinese) purely in virtue of executing an appropriate series of computations: what Searle coined 'Strong AI'.

Searle contends that understanding, of say a Chinese story, can never emerge purely as a result of following the procedures prescribed by any machine program, for in the CRA Searle offers a first-individual story delineating precisely how he could instantiate such a system, functioning as the Central Processing Unit of a computer, produce right internal and external state transitions, and even pass a 'Turing test' for comprehension of Chinese, and yet still not comprehend a single word of Chinese.

In Mind, Brains and Programs, Searle [33] states that in Strong AI "the computer is not merely a tool in the study of the mind; rather the appropriately programmed computer really is a mind, in the sense that, computers given the right programs can be literally said to understand and have other cognitive states".

Accordingly, in Strong Computational Creativity, we argue that computer is not merely a tool in the study of the creativity; rather the appropriately programmed computer really is creative, in the sense that, computers given the right programs can be literally said to understand its creation and have other cognitive states (e.g. teleological and emotive) associated with human creative processes.

In other words, the creative human fundamentally knows that $\mathrm{s} / \mathrm{he}$ is making a mark on paper (or a computer screen) or moulding clay or making sound etc. Whereas merely by following the rules of the program, the computer cannot know that it is doing anything other than manipulating uninterpreted symbols (or squiggles).

We fundamentally suggest that Searle's famous thought experiment also targets the analogous notion of 'strong computational creativity'. I.e. Searle using a similar "room" could get so good at following the rules that the strings of symbols he outputs from the room successfully control a 'Strong' computationally controlled creative art-system, producing works judged to have artistic merit by people outside the room, even though Searle-in-the-room remains ignorant of the produced art and the externally labelled 'art practise'. To paraphrase Dennett's deployment of 'the intentional stance', the computational system is merely instantiating a form of 'as-if creativity' without any real cognitive states, meaning or intentionality. I.e. Any 'creativity' ascribed to the computational system is merely a reflection of the engineer that designed it and the user who operated it in a given social nexus.

\section{The body in question}

In our opinion Searle's Chinese room argument suggests that to take the notion of 'Strong creativity' seriously we need to move away from purely computational ex- 
planations of creativity and look at how human meaning and creative processes are fundamentally grounded in the human body and society; taking the body, issues of embodiment and our social embedding, much more seriously. And this 'strong' notion of embodiment cannot simply be realised by opting a putative computationally creative system onto a conventional 'tin can robot' 1 .

As Nasuto, Bishop et al. [28] a fortiori argue in their discussion of Biologically controlled animats $^{2}$ and the so-called 'Zombie' animals ${ }^{3}$ (two examples carefully chosen to lie at polar ends of the spectrum of possible engineered robotic/cyborg systems), merely instantiating appropriate sensorimotor coupling is not sufficient to instantiate meaningful intentional states, as in both cases the induced behavioural couplings are not the effect of the intrinsic nervous system's constraints (metabolic or otherwise) at any level. On the contrary, they are actually the cause of extrinsic metabolic demands (made via the experimenter's externally directed manipulations). Since the experimenter drives the sensorimotor couplings in an arbitrary way (from the perspective of the intrinsic metabolic needs of animal or its cellular constituents), the causal relationship between the bodily milieu and the motor actions and sensory readings can never be genuinely and appropriately coupled. Thus Nasuto \& Bishop [28] assert that only the 'right type' and 'directionality' of sensorimotor couplings can ultimately lead to genuine understanding and intentionality.

For example, considering the case of the optogentically remotely controlled rat, even though the animal still possesses a fully functional biological body (and, arguably, a functioning brain), the fact that it receives external [optogenetic] commands cannot give rise to a genuine understanding of what the animal is experiencing; the rat's brain receives alien input that, at best, may resemble drug-induced decontextualised hallucinations - mere uninterpreted symbols/squiggles and squoggles - which will remain meaningless despite how accustomed the animal may become to this new mode of remotely induced behaviour. The situation is analogous to the alien hand syndrome - where in patients, for example, see their arm perform actions not of their volition or under their control. Such patients never ascribe meaning as to why their arm acted in this way, albeit they can see (and hence comprehend) the actions in the same way as any other observer; in this sense they are 'external observers' of their own limb(s) 'zombie movements'.

In contrast we suggest that fully intentional creative acts, as engaged in the process of genuinely understanding the world, require both a fully functional brain and a fully functional body; so translating the question of the requirements for 'genuine creative thinking' to the defining features of the processes whereby the brain and body interact with the world and society.

\footnotetext{
${ }^{1}$ Whereby a robot body is imply bolted on to an an appropriate AI and the material of that 'embodiment' is effectively unimportant.

${ }^{2}$ Robots controlled by a cultured-array of real biological neurons.

${ }^{3}$ E.g. An animal whose behaviour is 'remotely-controlled', by an external experimenter, say by optogenetics; see also Gradinaru et al [20], who used optogenetic techniques to stimulate neurons selectively, inducing motor behaviour without requiring conditioning.
} 
In the light of these concerns, until the challenge of the CRA has been fully met, we urge caution in ascribing 'Strong' notions of creativity to any fundamentally computational system.

\section{Conclusion}

In this paper, we have discussed the potential of the swarms in exhibiting 'weak computational creativity'. This specific work described herein uses swarm intelligence techniques to explore the difference between using Random Freedom and Swarmic Freedom in the visualisation of the swarms 'tracing' line drawings; this work highlights the features of swarm-regulated difference versus simple-random difference in the production of such 'sketches' by computer. We stressed on the significant impact of both freedom and constraint on the emergent creativity, and presented a discussion on how these two concepts are mapped onto exploration and exploitation, two of the most infamous phases in the swarm intelligence world.

The 'weak computationally creative' artist described herein is the result of merging two swarm intelligence algorithms, preserving freedom (exploration) and constraint (exploitation).

The Strong computational creativity is presented in ways emphasising:

- that genuine creativity implies 'genuine understanding' and other cognitive states.

- autonomy, asserting that without 'Strong Embodiment', computational systems are not genuinely autonomous.

Therefore, we urge caution in ascribing "Strong creativity" to any merely computational system.

\section{References}

1. al-Rifaie, M.M., Bishop, M.: Stochastic diffusion search review. In: Paladyn, Journal of Behavioral Robotics, vol. 4, pp. 155-173. Paladyn, Journal of Behavioral Robotics (2013). DOI 10.2478/pjbr-2013-0021

2. al-Rifaie, M.M., Bishop, M., Aber, A.: Creative or not? birds and ants draw with muscles. In: AISB 2011: Computing and Philosophy, pp. 23-30. University of York, York, U.K. (2011). ISBN: 978-1-908187-03-1

3. al-Rifaie, M.M., Bishop, M., Blackwell, T.: An investigation into the use of swarm intelligence for an evolutionary algorithm optimisation. In: International Conference on Evolutionary Computation Theory and Application (ECTA 2011). IJCCI (2011)

4. al-Rifaie, M.M., Bishop, M., Blackwell, T.: Information sharing impact of stochastic diffusion search on differential evolution algorithm. J. Memetic Computing 4(4), 327-338 (2012). DOI 10.1007/s12293-012-0094-y. URL http://dx.doi.org/10.1007/s12293-012-0094-y

5. al-Rifaie, M.M., Bishop, M., Blackwell, T.: Resource allocation and dispensation impact of stochastic diffusion search on differential evolution algorithm. In: D. Pelta, 
N. Krasnogor, D. Dumitrescu, C. Chira, R. Lung (eds.) Nature Inspired Cooperative Strategies for Optimization (NICSO 2011), Studies in Computational Intelligence, vol. 387, pp. 21-40. Springer Berlin Heidelberg (2012). DOI 10.1007/978-3-642-24094-2_2. URL http://dx.doi.org/10.1007/978-3-642-24094-2_2

6. al-Rifaie, M.M., Bishop, M., Caines, S.: Creativity and autonomy in swarm intelligence systems. J. Cognitive Computation 4(3), 320-331 (2012). DOI 10.1007/s12559-012-9130-y. URL http://dx.doi.org/10.1007/s12559-012-9130-y

7. al-Rifaie, M.M., Bishop, M.J., Blackwell, T.: An investigation into the merger of stochastic diffusion search and particle swarm optimisation. In: Proceedings of the 13th annual conference on Genetic and evolutionary computation, GECCO '11, pp. 3744. ACM, New York, NY, USA (2011). DOI 10.1145/2001576.2001583. URL http://doi.acm.org/10.1145/2001576.2001583

8. Aupetit, S., Bordeau, V., Monmarche, N., Slimane, M., Venturini, G.: Interactive evolution of ant paintings. In: The 2003 Congress on Evolutionary Computation, 2003. CEC'03., vol. 2, pp. 1376-1383 (2004)

9. Boden, M.: Creativity and Art: Three Roads to Surprise. Oxford University Press (2010)

10. Borgia, G.: Complex male display and female choice in the spotted bowerbird: specialized functions for different bower decorations. Animal Behaviour 49, 1291-1301 (1995)

11. Bown, O.: Generative and adaptive creativity. In: J. McCormack, M. d'Inverno (eds.) In Computers and Creativity. Berlin: Springer (2011)

12. Callan, R.: Artificial Intelligence. Palgrave Macmillan (2003)

13. Clark, A.: Natural-born cyborgs: Minds, technologies, and the future of human intelligence. Oxford University Press (2003)

14. Collomosse, J., Hall, P.: Genetic paint: A search for salient paintings. Applications of Evolutionary Computing pp. 437-447 (2005)

15. Curtis, C.J.: Loose and sketchy animation. In: ACM SIGGRAPH 98 Electronic art and animation catalog, p. 145 (1998)

16. Dorin, A., Korb, K.: Creativity refined. in computers and creativity. In: J. McCormack, M. d'Inverno (eds.) In Computers and Creativity. Berlin: Springer (2011)

17. Eberhart, R., Kennedy, J.: A new optimizer using particle swarm theory. In: Proceedings of the sixth international symposium on micro machine and human science, vol. 43. New York, NY, USA: IEEE (1995)

18. Etzioni, A., Ben-Barak, A., Peron, S., Durandy, A.: Ataxia-telangiectasia in twins presenting as autosomal recessive hyper-immunoglobulin m syndrome. IMAJ 9(5), 406 (2007)

19. Galanter, P.: Computational aesthetic evaluation: Past and future. In: J. McCormack, M. d'Inverno (eds.) In Computers and Creativity. Berlin: Springer (2011)

20. Gradinaru, V., Thompson, K.R., Zhang, F., Mogri, M., Kay, K., Schneider, M.B., Deisseroth, K.: Targeting and readout strategies for fast optical neural control in vitro and in vivo. J Neurosci 26:27(52), 14,231-14,238 (2007)

21. Greenfield, G.: Evolutionary methods for ant colony paintings. APPLICATIONS OF EVOLUTIONARY COMPUTING, PROCEEDINGS 3449, 478-487 (2005)

22. Hertzmann, A.: Paint by relaxation. In: Computer Graphics International 2001. Proceedings, pp. 47-54. IEEE (2001)

23. Johnson-Laird, P.N.: Freedom and constraint in creativity. In: R.J. Sternberg (ed.) The nature of creativity: contemporary psychological perspectives, p. 202219. Cambridge University Press (1988)

24. Kennedy, J., Eberhart, R.C.: Particle swarm optimization. In: Proceedings of the IEEE International Conference on Neural Networks, vol. IV, pp. 1942-1948. IEEE Service Center, Piscataway, NJ (1995)

25. Monmarche, N., Aupetit, S., Bordeau, V., Slimane, M., Venturini, G.: Interactive evolution of ant paintings. In: B.M.e. al (ed.) 2003 Congress on Evolutionary Computation, vol. 2, pp. 1376-1383. IEEE Press (2003)

26. Moura, L., Ramos, V.: Swarm paintings-nonhuman art. ARCHITOPIA book, art, architecture and science pp. 5-24 (2007) 
27. Nagel, T.: What is it like to be a bat? The Philosophical Review 83(4), 435-450 (1974)

28. Nasuto, S., Bishop, J., Roesch, E., Spencer, M.: Zombie mouse in a chinese room. Philosophy \& Technology pp. 1-15 (2014). DOI 10.1007/s13347-014-0150-2. URL http://dx.doi.org/10.1007/s13347-014-0150-2

29. Preston, J., Bishop, M.: Views into the Chinese room: New essays on Searle and artificial intelligence. Oxford University Press (2002)

30. Restany, P.: Hundertwasser: the painter-king with the five skins: the power of art. Taschen America Llc (2001)

31. Rothenberg, A., Hausman, C.: The creativity question. Duke University Press Books (1976)

32. Schlechtweg, S., Germer, T., Strothotte, T.: Renderbots-multi-agent systems for direct image generation. In: Computer Graphics Forum, vol. 24, pp. 137-148 (2005)

33. Searle, J.: Minds, brains, and programs. Behavioral and Brain Sciences 3(3), 417-457 (1980)

34. Semet, Y., O'Reilly, U.M., Durand, F.: An interactive artificial ant approach to nonphotorealistic rendering. In: Genetic and Evolutionary Computation-GECCO 2004, pp. 188 200 (2004)

35. Sternberg, R.: The nature of creativity: Contemporary psychological perspectives. Cambridge Univ $\operatorname{Pr}(1988)$

36. Turk, G., Banks, D.: Image-guided streamline placement. In: Proceedings of the 23rd annual conference on Computer graphics and interactive techniques, pp. 453-460. ACM (1996)

37. Urbano, P.: Playing in the pheromone playground: Experiences in swarm painting. Applications on Evolutionary Computing pp. 527-532 (2005)

38. Urbano, P.: Consensual paintings. Applications of Evolutionary Computing pp. 622-632 (2006)

39. Watanabe, S.: Pigeons can discriminate "good" and "bad" paintings by children. Animal Cognition 13(1) (2009)

40. Weesatchanam, A.M.: Are Paintings by Elephants Really Art? The Elephant Art Gallery (2006)

41. Zhao, M., Zhu, S.: Customizing painterly rendering styles using stroke processes. In: Proceedings of the ACM SIGGRAPH/Eurographics Symposium on Non-Photorealistic Animation and Rendering, pp. 137-146. ACM (2011) 